\title{
On the solutions and conservation laws of the coupled Drinfeld-Sokolov-Satsuma-Hirota system
}

\author{
Khadijo Rashid Adem ${ }^{\dagger}$ and Chaudry Masood Khalique ${ }^{* \dagger}$
}

\author{
*Correspondence: \\ Masood.Khalique@nwu.ac.za \\ Department of Mathematical \\ Sciences, International Institute for \\ Symmetry Analysis and \\ Mathematical Modelling, \\ North-West University, Mafikeng \\ Campus, Private Bag X 2046, \\ Mmabatho, 2735, Republic of South \\ Africa \\ ${ }^{\dagger}$ Equal contributors
}

\begin{abstract}
In this paper we study the coupled Drinfeld-Sokolov-Satsuma-Hirota system, which was developed as one example of nonlinear equations possessing Lax pairs of a special form. Also this system was found as a special case of the four-reduction of the Kadomtsev-Petviashivilli hierarchy. We obtain exact solutions of the system by using Lie symmetry analysis along with the simplest equation and Jacobi elliptic equation methods. Also, symmetry reductions are obtained based on the optimal system of one-dimensional subalgebras. In addition, the conservation laws are derived using two approaches: the new conservation theorem due to Ibragimov and the multiplier method.

Keywords: coupled Drinfeld-Sokolov-Satsuma-Hirota system; Lie symmetry methods; simplest equation method; Jacobi elliptic function method; conservation laws
\end{abstract}

\section{Introduction}

In recent years many nonlinear evolution equations (NLEEs) have been used to model many real world problems in various fields of science and engineering. Thus, finding exact explicit solutions of NLEEs is a very important endeavor. It is also true that finding solutions of NLEEs is a difficult task, and only in few special cases one can write down the explicit solutions. However, despite of this fact, various methods of solving NLEEs have been proposed in the literature recently. Some of the most important methods found in the literature include the ansatz method $[1,2]$, the Weierstrass elliptic function expansion method [3], the Darboux transformation [4], Hirota's bilinear method [5], the $\left(G^{\prime} / G\right)$-expansion method [6], the Jacobi elliptic function expansion method [7, 8], the inverse scattering transform method [9], the homogeneous balance method [10], the Bäcklund transformation [11], the F-expansion method [12], the exp-function method [13], the multiple expfunction method [14], the variable separation approach [15], the sine-cosine method [16], the tri-function method [17, 18], and the Lie symmetry method [19-25].

In this paper we study the coupled Drinfeld-Sokolov-Satsuma-Hirota (DSSH) system

$$
\begin{aligned}
& u_{t}-6 u u_{x}+u_{x x x}-6 v_{x}=0, \\
& v_{t}-2 v_{x x x}+6 u v_{x}=0 .
\end{aligned}
$$

@ 2014 Adem and Khalique; licensee Springer. This is an Open Access article distributed under the terms of the Creative Commons Attribution License (http://creativecommons.org/licenses/by/4.0), which permits unrestricted use, distribution, and reproduction in any medium, provided the original work is properly credited. 
This system was introduced independently by Drinfeld and Sokolov [26], and by Satsuma and Hirota [27]. The coupled DSSH system [26] was given as one of numerous examples of nonlinear equations possessing Lax pairs of a special form. Also, the coupled DSSH system [27] was found as a special case of the four-reduction of the KP hierarchy, and its explicit one-soliton solution was constructed. Gürses and Karasu [28] found a recursion operator and a bi-Hamiltonian structure for (1). Wazwaz [29] used three distinct methods, namely the Cole-Hopf transformation, Hirota's bilinear and the exp-function methods, and obtained solitons, multiple soliton solutions, multiple singular soliton solutions, and plane periodic solutions. Zheng [30] used the $\left(G^{\prime} / G\right)$-expansion method and obtained traveling wave solutions of (1).

In this paper we firstly perform symmetry reductions of (1) using Lie group analysis [19$24]$, which are based on the optimal systems of one-dimensional subalgebras. The simplest equation method [31] and the Jacobi elliptic function method [32] are later employed to obtain some exact solutions of (1). In addition to this, conservation laws are derived for (1) using the new conservation theorem [33] and the multiplier method [34].

It is well known that the conservation laws play a very important role in the solution process of differential equations. Also, one can safely say that the existence of a large number of conservation laws of a system of partial differential equations is a strong indication of its integrability [19]. Recently, conservation laws have been used to find exact solutions of certain partial differential equations $[35,36]$.

\section{Symmetry analysis of (1)}

The symmetry group of the coupled DSSH system (1) will be generated by the vector field of the form

$$
X=\xi^{1}(t, x, u, v) \frac{\partial}{\partial t}+\xi^{2}(t, x, u, v) \frac{\partial}{\partial x}+\eta^{1}(t, x, u, v) \frac{\partial}{\partial u}+\eta^{2}(t, x, u, v) \frac{\partial}{\partial v} .
$$

The application of the third prolongation $\mathrm{pr}^{(3)} X$ to (1) results in an overdetermined system of linear partial differential equations. The general solution of these equations with the aid of Maple is given by

$$
\begin{aligned}
& \xi^{1}(t, x, u, v)=C_{1}+3 t C_{4}, \\
& \xi^{2}(t, x, u, v)=C_{2}+x C_{4}, \\
& \eta^{1}(t, x, u, v)=-2 C_{4} u, \\
& \eta^{2}(t, x, u, v)=C_{3}-4 v C_{4},
\end{aligned}
$$

where $C_{i}, i=1, \ldots, 4$, are arbitrary constants. The above general solution contains four arbitrary constants, and hence the infinitesimal symmetries of (1) form the four-dimensional Lie algebra spanned by the following linearly independent operators:

$$
X_{1}=\frac{\partial}{\partial t}, \quad X_{2}=\frac{\partial}{\partial x}, \quad X_{3}=\frac{\partial}{\partial v}, \quad X_{4}=3 t \frac{\partial}{\partial t}+x \frac{\partial}{\partial x}-2 u \frac{\partial}{\partial u}-4 v \frac{\partial}{\partial v} .
$$

\subsection{Optimal system of one-dimensional subalgebras}

In this subsection we present the optimal system of one-dimensional subalgebras for equation (1) to obtain the optimal system of group-invariant solutions. The method which we 
Table 1 Commutator table of the Lie algebra of equation (1)

\begin{tabular}{lllll}
\hline & $\boldsymbol{X}_{\mathbf{1}}$ & $\boldsymbol{X}_{\mathbf{2}}$ & $\boldsymbol{X}_{\mathbf{3}}$ & $\boldsymbol{X}_{\mathbf{4}}$ \\
\hline$X_{1}$ & 0 & 0 & 0 & $3 X_{2}$ \\
$X_{2}$ & 0 & 0 & 0 & $X_{2}$ \\
$X_{3}$ & 0 & 0 & 0 & $-2 X_{3}$ \\
$X_{4}$ & $-3 X_{2}$ & $-X_{2}$ & $2 X_{3}$ & 0 \\
\hline
\end{tabular}

Table 2 Adjoint table of the Lie algebra of equation (1)

\begin{tabular}{lllll}
\hline Ad & $\boldsymbol{X}_{\mathbf{1}}$ & $\boldsymbol{X}_{\mathbf{2}}$ & $\boldsymbol{X}_{\mathbf{3}}$ & $\boldsymbol{X}_{\mathbf{4}}$ \\
\hline$X_{1}$ & $X_{1}$ & $X_{2}$ & $X_{3}$ & $-3 \varepsilon X_{1}+X_{4}$ \\
$X_{2}$ & $X_{1}$ & $X_{2}$ & $X_{3}$ & $-\varepsilon X_{2}+X_{4}$ \\
$X_{3}$ & $X_{1}$ & $X_{2}$ & $X_{3}$ & $4 \varepsilon X_{3}+X_{4}$ \\
$X_{4}$ & $3 e^{\varepsilon} X_{1}$ & $e^{\varepsilon} X_{2}$ & $e^{-4 \varepsilon} X_{3}$ & $X_{4}$ \\
\hline
\end{tabular}

use here for obtaining the optimal system of one-dimensional subalgebras is given in [20]. The adjoint transformations are given by

$$
\operatorname{Ad}\left(\exp \left(\varepsilon X_{i}\right)\right) X_{j}=X_{j}-\varepsilon\left[X_{i}, X_{j}\right]+\frac{1}{2} \varepsilon^{2}\left[X_{i},\left[X_{i}, X_{j}\right]\right]-\cdots
$$

The commutator table of the Lie point symmetries of equation (1) and the adjoint representations of the symmetry group of (1) on its Lie algebra are given in Table 1 and Table 2, respectively. Table 1 and Table 2 are then used to construct the optimal system of onedimensional subalgebras for equation (1).

From Tables 1 and 2 and following [20], one can obtain an optimal system of onedimensional subalgebras given by $\left\{X_{4}, b X_{1}+c X_{2}+d X_{3}\right\}$, where $b, c, d=0, \pm 1$.

\subsection{Symmetry reductions of (1)}

In this subsection we use the optimal system of one-dimensional subalgebras calculated above to obtain symmetry reductions.

Case $1 . X_{4}$

The operator $X_{4}$ gives rise to the group-invariant solution

$$
u=t^{-\frac{2}{3}} F(z), \quad v=t^{-\frac{4}{3}} G(z)
$$

where $z=x t^{-1 / 3}$ is an invariant of the symmetry $X_{4}$. Substitution of (2) into (1) results in the system of ordinary differential equations (ODEs), where $F$ and $G$ satisfy

$$
\begin{aligned}
& 4 G(z)+6 G^{\prime \prime \prime}(z)-18 F(z) G^{\prime}(z)+z G^{\prime}(z)=0, \\
& 2 F(z)-3 F^{\prime \prime \prime}(z)+z F(z)+18 F(z) F^{\prime}(z)+18 G(z)=0 .
\end{aligned}
$$

Case 2. $b X_{1}+c X_{2}+d X_{3} ; b, c, d=0, \pm 1$

The symmetry $b X_{1}+c X_{2}+d X_{3}$ gives rise to the group-invariant solution

$$
u=F(z), \quad v=G(z)+t d / b,
$$


where $z=(b x-c t) / b$ is an invariant of the symmetry $b X_{1}+c X_{2}+d X_{3}$. The insertion of (3) into (1) results in the system of ODEs

$$
\begin{aligned}
& 6 b F(z) F^{\prime}(z)+c F^{\prime}(z)-b F^{\prime \prime \prime}(z)+6 b G^{\prime}(z)=0, \\
& c G^{\prime}(z)-6 b F(z) G^{\prime}(z)+2 b G^{\prime \prime \prime}(z)-d=0 .
\end{aligned}
$$

\section{Exact solutions of (1) using the simplest equation method}

Taking the linear combination of the translation symmetries, viz. $X_{1}+\lambda X_{2}$, and solving the corresponding Lagrange system for the symmetry $X_{1}+\lambda X_{2}$, one obtains an invariant $z=x-\lambda t$ and the group-invariant solution of the form

$$
u=F(z), \quad v=G(z)
$$

where the functions $F$ and $G$ satisfy

$$
\begin{aligned}
& F^{\prime \prime \prime}(z)-\lambda F^{\prime}(z)-6 F(z) F^{\prime}(z)-6 G^{\prime}(z)=0, \\
& 6 F(z) G^{\prime}(z)-2 G^{\prime \prime \prime}(z)-\lambda G^{\prime}(z)=0 .
\end{aligned}
$$

Now we use the simplest equation method [25, 31] to solve system (5a)-(5b); and as a result, we obtain the exact solutions of our coupled DSSH system (1). Bernoulli and Riccati equations will be used as the simplest equations.

Let us consider the solutions of (5a)-(5b) in the form

$$
F(z)=\sum_{i=0}^{M} \mathcal{A}_{i}(H(z))^{i}, \quad G(z)=\sum_{i=0}^{N} \mathcal{B}_{i}(H(z))^{i},
$$

where $H(z)$ satisfies the Bernoulli or Riccati equation, $M$ and $N$ are positive integers that can be determined by a balancing procedure and $\mathcal{A}_{i}$ 's and $\mathcal{B}_{i}$ 's are parameters to be determined.

\subsection{Solutions of (1) using the Bernoulli equation as the simplest equation}

The balancing procedure gives $M=2$ and $N=4$, and hence the solutions of (5a)-(5b) are of the form

$$
\begin{aligned}
& F(z)=\mathcal{A}_{0}+\mathcal{A}_{1} H+\mathcal{A}_{2} H^{2}, \\
& G(z)=\mathcal{B}_{0}+\mathcal{B}_{1} H+\mathcal{B}_{2} H^{2}+\mathcal{B}_{3} H^{3}+\mathcal{B}_{4} H^{4} .
\end{aligned}
$$

Substituting (7a)-(7b) into (5a)-(5b) and making use of the Bernoulli equation [25] and then equating the coefficients of the functions $H^{i}$ to zero, we obtain an algebraic system of equations in terms of $\mathcal{A}_{i}(i=0,1,2)$ and $\mathcal{B}_{i}(i=0,1,2,3,4)$. Solving the resultant system of algebraic equations with the aid of Maple, one possible set of values of $\mathcal{A}_{i}$ and $\mathcal{B}_{i}$ is as follows:

$$
\lambda=\frac{7}{2} a^{2}, \quad \mathcal{A}_{0}=\frac{11}{12} a^{2}, \quad \mathcal{A}_{1}=10 a b, \quad \mathcal{A}_{2}=10 b^{2},
$$




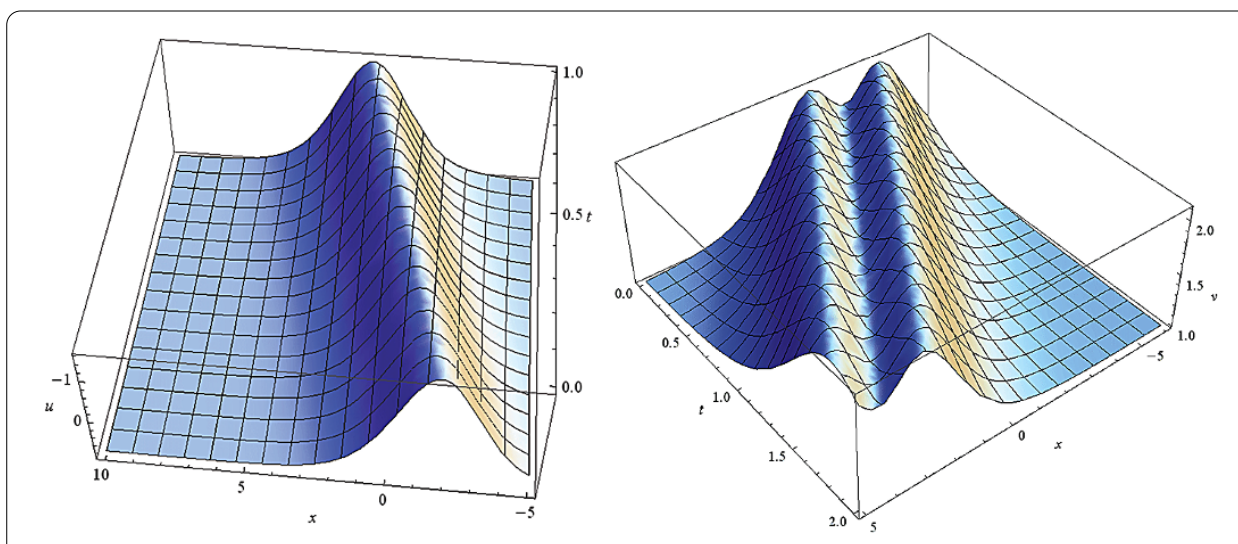

Figure 1 Profile of (8a)-(8b).

$$
\mathcal{B}_{1}=-\frac{40}{3} a^{3} b, \quad \mathcal{B}_{2}=-\frac{160}{3} a^{2} b^{2}, \quad \mathcal{B}_{3}=-80 b^{3} a, \quad \mathcal{B}_{4}=-40 b^{4} .
$$

As a result, a solution of (1) is

$$
\begin{aligned}
u(t, x)= & \mathcal{A}_{0}+\mathcal{A}_{1} a\left\{\frac{\cosh [a(z+C)]+\sinh [a(z+C)]}{1-b \cosh [a(z+C)]-b \sinh [a(z+C)]}\right\} \\
& +\mathcal{A}_{2} a^{2}\left\{\frac{\cosh [a(z+C)]+\sinh [a(z+C)]}{1-b \cosh [a(z+C)]-b \sinh [a(z+C)]}\right\}^{2}, \\
v(t, x)= & \mathcal{B}_{0}+\mathcal{B}_{1} a\left\{\frac{\cosh [a(z+C)]+\sinh [a(z+C)]}{1-b \cosh [a(z+C)]-b \sinh [a(z+C)]}\right\} \\
& +\mathcal{B}_{2} a^{2}\left\{\frac{\cosh [a(z+C)]+\sinh [a(z+C)]}{1-b \cosh [a(z+C)]-b \sinh [a(z+C)]}\right\}^{2} \\
& +\mathcal{B}_{3} a^{3}\left\{\frac{\cosh [a(z+C)]+\sinh [a(z+C)]}{1-b \cosh [a(z+C)]-b \sinh [a(z+C)]}\right\}^{3} \\
& +\mathcal{B}_{4} a^{4}\left\{\frac{\cosh [a(z+C)]+\sinh [a(z+C)]}{1-b \cosh [a(z+C)]-b \sinh [a(z+C)]}\right\}^{4},
\end{aligned}
$$

where $z=x-\lambda t$. The profile of solution (8a)-(8b) is given in Figure 1 .

\subsection{Solutions of ( 1 ) using the Riccati equation as the simplest equation}

In this case the balancing procedure also gives the same values of $M$ and $N$, i.e., $M=2$ and $N=4$. Thus the solutions of (5a)-(5b) are of the form

$$
\begin{aligned}
& F(z)=\mathcal{A}_{0}+\mathcal{A}_{1} H+\mathcal{A}_{2} H^{2}, \\
& G(z)=\mathcal{B}_{0}+\mathcal{B}_{1} H+\mathcal{B}_{2} H^{2}+\mathcal{B}_{3} H^{3}+\mathcal{B}_{4} H^{4} .
\end{aligned}
$$

Substituting (9a)-(9b) into (5a)-(5b) and making use of the Riccati equation [25], we obtain an algebraic system of equations in terms of $\mathcal{A}_{i}$ and $\mathcal{B}_{i}$. Solving the resultant system, one possible set of values is as follows:

$$
\lambda=-\frac{7}{200}\left(\frac{400 a^{3} c-A_{1}^{2}}{a^{2}}\right), \quad \mathcal{A}_{0}=\frac{7,600 a^{3} c+11 A_{1}^{2}}{1,200 a^{2}},
$$




$$
\begin{aligned}
& \mathcal{A}_{1}=10 a b, \quad \mathcal{A}_{2}=10 a^{2}, \\
& \mathcal{B}_{1}=-\frac{A_{1}\left(200 a^{3} c+A_{1}^{2}\right)}{75 a^{2}}, \quad \mathcal{B}_{2}=-\frac{8}{15} A_{1}^{2}-\frac{80}{3} a^{3} c, \\
& \mathcal{B}_{3}=-8 a^{2} A_{1}, \quad \mathcal{B}_{4}=-40 a^{4} .
\end{aligned}
$$

Hence solutions of (1) are

$$
\begin{aligned}
u(t, x)= & \mathcal{A}_{0}+\mathcal{A}_{1}\left\{-\frac{b}{2 a}-\frac{\theta}{2 a} \tanh \left[\frac{1}{2} \theta(z+C)\right]\right\} \\
& +\mathcal{A}_{2}\left\{-\frac{b}{2 a}-\frac{\theta}{2 a} \tanh \left[\frac{1}{2} \theta(z+C)\right]\right\}^{2} \\
v(t, x)= & \mathcal{B}_{0}+\mathcal{B}_{1}\left\{-\frac{b}{2 a}-\frac{\theta}{2 a} \tanh \left[\frac{1}{2} \theta(z+C)\right]\right\} \\
& +\mathcal{B}_{2}\left\{-\frac{b}{2 a}-\frac{\theta}{2 a} \tanh \left[\frac{1}{2} \theta(z+C)\right]\right\}^{2} \\
& +\mathcal{B}_{3}\left\{-\frac{b}{2 a}-\frac{\theta}{2 a} \tanh \left[\frac{1}{2} \theta(z+C)\right]\right\}^{3} \\
& +\mathcal{B}_{4}\left\{-\frac{b}{2 a}-\frac{\theta}{2 a} \tanh \left[\frac{1}{2} \theta(z+C)\right]\right\}^{4}
\end{aligned}
$$

and

$$
\begin{aligned}
u(t, x)= & \mathcal{A}_{0}+\mathcal{A}_{1}\left\{-\frac{b}{2 a}-\frac{\theta}{2 a} \tanh \left(\frac{1}{2} \theta z\right)+\frac{\operatorname{sech}\left(\frac{\theta z}{2}\right)}{C \cosh \left(\frac{\theta z}{2}\right)-\frac{2 a}{\theta} \sinh \left(\frac{\theta z}{2}\right)}\right\} \\
& +\mathcal{A}_{2}\left\{-\frac{b}{2 a}-\frac{\theta}{2 a} \tanh \left(\frac{1}{2} \theta z\right)+\frac{\operatorname{sech}\left(\frac{\theta z}{2}\right)}{C \cosh \left(\frac{\theta z}{2}\right)-\frac{2 a}{\theta} \sinh \left(\frac{\theta z}{2}\right)}\right\}^{2} \\
v(t, x)= & \mathcal{B}_{0}+\mathcal{B}_{1}\left\{-\frac{b}{2 a}-\frac{\theta}{2 a} \tanh \left(\frac{1}{2} \theta z\right)+\frac{\operatorname{sech}\left(\frac{\theta z}{2}\right)}{C \cosh \left(\frac{\theta z}{2}\right)-\frac{2 a}{\theta} \sinh \left(\frac{\theta z}{2}\right)}\right\} \\
& +\mathcal{B}_{2}\left\{-\frac{b}{2 a}-\frac{\theta}{2 a} \tanh \left(\frac{1}{2} \theta z\right)+\frac{\operatorname{sech}\left(\frac{\theta z}{2}\right)}{C \cosh \left(\frac{\theta z}{2}\right)-\frac{2 a}{\theta} \sinh \left(\frac{\theta z}{2}\right)}\right\}^{2} \\
& +\mathcal{B}_{3}\left\{-\frac{b}{2 a}-\frac{\theta}{2 a} \tanh \left(\frac{1}{2} \theta z\right)+\frac{\operatorname{sech}\left(\frac{\theta z}{2}\right)}{C \cosh \left(\frac{\theta z}{2}\right)-\frac{2 a}{\theta} \sinh \left(\frac{\theta z}{2}\right)}\right\}^{3} \\
& +\mathcal{B}_{4}\left\{-\frac{b}{2 a}-\frac{\theta}{2 a} \tanh \left(\frac{1}{2} \theta z\right)+\frac{\operatorname{sech}\left(\frac{\theta z}{2}\right)}{C \cosh \left(\frac{\theta z}{2}\right)-\frac{2 a}{\theta} \sinh \left(\frac{\theta z}{2}\right)}\right\}^{4}
\end{aligned}
$$

where $z=x-\lambda t$.

\subsection{Solutions of (1) in terms of Jacobi elliptic functions}

We now present exact solutions of the coupled DSSH system (1) that are expressed in Jacobi elliptic functions. The cosine-amplitude function $\mathrm{cn}(z \mid \omega)$ and the sine-amplitude function $\operatorname{sn}(z \mid \omega)$ satisfy the first-order differential equations

$$
H^{\prime}(z)=-\left\{\left(1-H^{2}(z)\right)\left(1-\omega+\omega H^{2}(z)\right)\right\}^{\frac{1}{2}}
$$




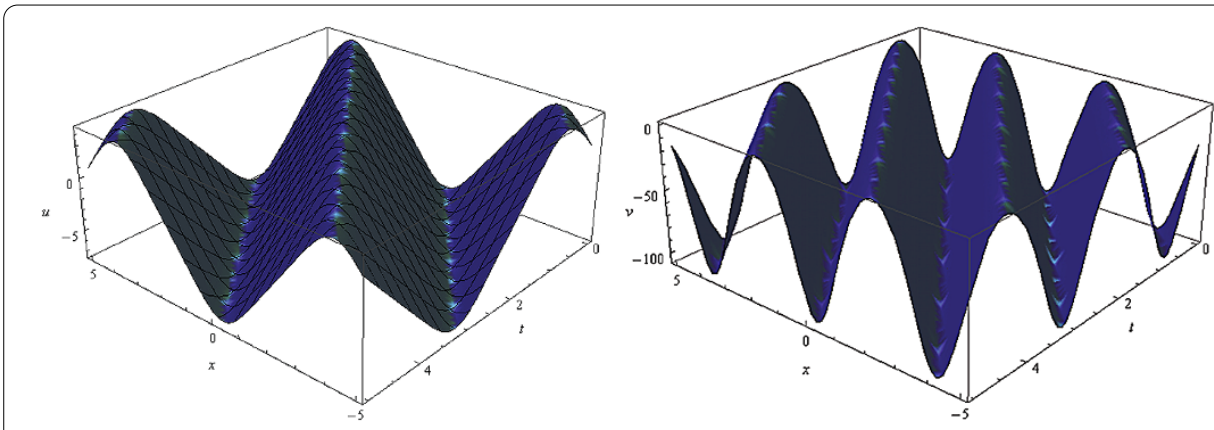

Figure 2 Profile of cniodal waves (14a)-(14b).

and

$$
H^{\prime}(z)=\left\{\left(1-H^{2}(z)\right)\left(1-\omega H^{2}(z)\right)\right\}^{\frac{1}{2}},
$$

respectively [32, 37].

Treating the above first-order ODEs as our simplest equations and then proceeding as before, we obtain the cnoidal and snoidal wave solutions that are given by

$$
\begin{aligned}
& u(t, x)=A_{0}+A_{1} \operatorname{cn}(z \mid \omega)+A_{2} \operatorname{cn}^{2}(z \mid \omega), \\
& v(t, x)=B_{0}+B_{1} \operatorname{cn}(z \mid \omega)+B_{2} \operatorname{cn}^{2}(z \mid \omega)+B_{3} \operatorname{cn}^{3}(z \mid \omega)+B_{4} \operatorname{cn}^{4}(z \mid \omega),
\end{aligned}
$$

where

$$
\begin{aligned}
& \lambda=42 k-280 \omega+140, \quad A_{0}=k, \quad A_{1}=-10 \omega, \quad A_{2}=0, \\
& B_{1}=0, \quad B_{2}=80 k \omega-480 \omega^{2}+240 \omega, \quad B_{3}=0, \quad B_{4}=-40 \omega^{2},
\end{aligned}
$$

$k$ is any root of $3 k^{2}+(-40 \omega+20) k+133 \omega^{2}-133 \omega+33 \omega=0$

and

$$
\begin{aligned}
& u(t, x)=A_{0}+A_{1} \operatorname{sn}(z \mid \omega)+A_{2} \operatorname{sn}^{2}(z \mid \omega), \\
& v(t, x)=B_{0}+B_{1} \operatorname{sn}(z \mid \omega)+B_{2} \operatorname{sn}^{2}(z \mid \omega)+B_{3} \operatorname{sn}^{3}(z \mid \omega)+B_{4} \operatorname{sn}^{4}(z \mid \omega),
\end{aligned}
$$

where

$$
\begin{aligned}
& \lambda=42 k+140 \omega+140, \quad A_{0}=k(v+4 \omega+4), \quad A_{1}=0, \quad A_{2}=10 \omega, \\
& B_{1}=0, \quad B_{2}=-80 k \omega-240 \omega^{2}-240 \omega, \quad B_{3}=0, \quad B_{4}=-40 \omega^{2},
\end{aligned}
$$

$k$ is any root of $3 k^{2}+(20 \omega+20) k+33 \omega^{2}+67 \omega+33=0$,

and $z=x-v t$. The profile of solution (14a)-(14b) is given in Figure 2.

\section{Conservation laws}

In this section we construct conservation laws for the coupled Drinfeld-Sokolov-SatsumaHirota system (1). The new conservation theorem due to Ibragimov [33] and the multiplier 
method [34] will be used. For the notations used in this section, the reader is referred to [33].

\subsection{Construction of conservation laws using the new conservation theorem}

In this subsection we construct conservation laws for (1) by applying the new conservation theorem [33].

The coupled DSSH system together with its adjoint equation is given by

$$
\begin{aligned}
& E_{\alpha_{1}} \equiv u_{t}-6 u u_{x}+u_{x x x}-6 v_{x}=0, \\
& E_{\alpha_{2}} \equiv v_{t}-2 v_{x x x}+6 u v_{x}=0, \\
& E_{\alpha_{1}}^{*} \equiv-p_{t}+6 u p_{x}-p_{x x x}+6 q v_{x}=0, \\
& E_{\alpha_{2}}^{*} \equiv-q_{t}-6 u q_{x}+2 q_{x x x}+6 p_{x}-6 q u_{x}=0 .
\end{aligned}
$$

One can easily verify that the third-order Lagrangian for the system of equations (15) and (16) is given by

$$
L=p\left(u_{t}-6 u u_{x}+u_{x x x}-6 v_{x}\right)+q\left(v_{t}-2 v_{x x x}+6 u v_{x}\right) .
$$

We recall that the coupled DSSH system admits the following four Lie point symmetries:

$$
X_{1}=\frac{\partial}{\partial t}, \quad X_{2}=\frac{\partial}{\partial x}, \quad X_{3}=\frac{\partial}{\partial v}, \quad X_{4}=3 t \frac{\partial}{\partial t}+x \frac{\partial}{\partial x}-2 u \frac{\partial}{\partial u}-4 v \frac{\partial}{\partial v} .
$$

Thus we have the following four cases:

(i) For the Lie point symmetry $X_{1}=\partial_{t}$, the corresponding Lie characteristic functions are $W^{1}=-u_{t}$ and $W^{2}=-v_{t}$. Thus, by using Ibragimov's theorem [33], the components of the conserved vector are given by

$$
\begin{aligned}
& T_{1}^{t}=-6 p u_{x} u+p u_{x x x}-6 p v_{x}+6 q u v_{x}-2 q v_{x x x}, \\
& T_{1}^{x}=6 p u u_{t}-p u_{t x x}+6 p v_{t}-6 q u v_{t}+2 q v_{t x x}-u_{t} p_{x x}+p_{x} u_{t x}+2 v_{t} q_{x x}-2 q_{x} v_{t x} .
\end{aligned}
$$

(ii) The Lie point symmetry $X_{2}=\partial_{x}$ has the Lie characteristic functions that are given by $W^{1}=-u_{x}$ and $W^{2}=-v_{x}$. Hence, by the application of Ibragimov's theorem [33], the conserved vector $\left(T_{2}^{t}, T_{2}^{x}\right)$ is given by

$$
\begin{aligned}
& T_{2}^{t}=-p u_{x}-q v_{x}, \\
& T_{2}^{x}=p u_{t}+q v_{t}-u_{x} p_{x x}+p_{x} u_{x x}+2 v_{x} q_{x x}-2 q_{x} v_{x x} .
\end{aligned}
$$

(iii) The symmetry generator $X_{3}=\partial_{\nu}$ has the Lie characteristic functions given by $W^{1}=0$ and $W^{2}=1$, and hence in this case one can obtain the conserved vector whose components are

$$
\begin{aligned}
& T_{3}^{t}=q, \\
& T_{3}^{x}=-6 p+6 u q-2 q_{x x} .
\end{aligned}
$$


(iv) Finally, we consider the symmetry generator $X_{4}=-3 t \partial_{t}-x \partial_{x}+2 u \partial_{u}+4 v \partial_{v}$, which has the Lie characteristic functions $W^{1}=2 u+3 t u_{t}+x u_{x}$ and $W^{2}=4 v+3 t v_{t}+x v_{x}$. By invoking Ibragimov's theorem [33], the components of the conserved vector are given by

$$
\begin{aligned}
T_{4}^{t}= & 18 t p u u_{x}+x p u_{x}-3 t p u_{x x x}+18 t p v_{x}-18 t q u v_{x}+x q v_{x}+6 t q v_{x x x}+2 p u+4 q v, \\
T_{4}^{x}= & 2 u p_{x x}-18 t p u u_{t}+4 p u_{x x}-x p u_{t}+3 t p u_{t x x}-18 t p v_{t}+18 t q u v_{t}-8 v q_{x x}-12 q v_{x x} \\
& -x q v_{t}-6 t q v_{t x x}-12 u^{2} p-24 p v+24 q u v+3 t u_{t} p_{x x}-3 t p_{x} u_{t x}-3 p_{x} u_{x}+x u_{x} p_{x x} \\
& -x p_{x} u_{x x}-6 t v_{t} q_{x x}+6 t q_{x} v_{t x}+10 q_{x} v_{x}-2 x v_{x} q_{x x}+2 x q_{x} v_{x x} .
\end{aligned}
$$

\subsection{Construction of conservation laws using the multiplier method}

Here we use the multiplier method [34] to construct conservation laws for the coupled DSSH system (1). The second-order multipliers $\Lambda_{1}=\Lambda_{1}\left(t, x, u, v, u_{x}, v_{x}, u_{x x}, v_{x x}\right)$ and $\Lambda_{2}=$ $\Lambda_{2}\left(t, x, u, v, u_{x}, v_{x}, u_{x x}, v_{x x}\right)$ are given by

$$
\begin{aligned}
& \Lambda_{1}=\frac{1}{2}\left(3 u^{2}-u_{x x}+2 v\right) C_{2}+C_{1} t u+C_{3} u+\frac{1}{6} C_{1} x, \\
& \Lambda_{2}=C_{2} u+C_{1} t+C_{3},
\end{aligned}
$$

where $C_{i}, i=1, \ldots, 3$, are arbitrary constants. Corresponding to the above multipliers, we obtain the following three local conserved vectors of (1):

$$
\begin{aligned}
& T_{1}^{t}=\frac{1}{6}\left\{3 t u^{2}+x u+6 t v\right\} \\
& T_{1}^{x}=\frac{1}{6}\left\{6 t u_{x x} u-12 t u^{3}-3 x u^{2}-6 x v-3 t u_{x}^{2}-12 t v_{x x}-u_{x}+x u_{x x}\right\} \\
& T_{2}^{t}=\frac{1}{4}\left\{-u_{x x} u+4 u v+2 u^{3}\right\} \\
& T_{2}^{x}=\frac{1}{4}\left\{-8 v_{x x} u+4 u_{x x} v+6 u_{x x} u^{2}+u u_{t x}-12 u^{2} v-9 u^{4}-12 v^{2}-u_{t} u_{x}+8 u_{x} v_{x}-u_{x x}^{2}\right\}
\end{aligned}
$$

and

$$
\begin{aligned}
& T_{3}^{t}=\frac{1}{2}\left\{u^{2}+2 v\right\}, \\
& T_{3}^{x}=\frac{1}{2}\left\{2 u_{x x} u-4 u^{3}-u_{x}^{2}-4 v_{x x}\right\} .
\end{aligned}
$$

Remark It should be noted that higher-order conservation laws of (1) can be computed by increasing the order of the multipliers.

\section{Concluding remarks}

In this paper firstly we obtained the solutions of the Drinfeld-Sokolov-Satsuma-Hirota equation by employing Lie group analysis together with the simplest and Jacobi elliptic equation methods. Also symmetry reductions were obtained based on the optimal systems of one-dimensional subalgebras. The exact solutions obtained were traveling wave solutions, cnoidal and snoidal wave solutions. Furthermore, the conservation laws for the 
underlying equation were derived by using two different approaches, namely the new conservation theorem and the multiplier method. The importance of the conservation laws was explained in the introduction.

\section{Competing interests}

The authors declare that they have no competing interests.

\section{Authors' contributions}

KRA and CMK worked together in the derivation of the mathematical results. Both authors read and approved the final manuscript.

\section{Acknowledgements}

KRA is grateful to the National Research Foundation of South Africa for the generous financial support.

Received: 18 September 2014 Accepted: 13 November 2014 Published online: 09 December 2014

\section{References}

1. $\mathrm{Hu}, \mathrm{JL}$ : Explicit solutions to three nonlinear physical models. Phys. Lett. A 287, 81-89 (2001)

2. $\mathrm{Hu}, \mathrm{JL}$ : A new method for finding exact traveling wave solutions to nonlinear partial differential equations. Phys. Lett. A 286, 175-179 (2001)

3. Chen, Y, Yan, Z: The Weierstrass elliptic function expansion method and its applications in nonlinear wave equations. Chaos Solitons Fractals 29, 948-964 (2006)

4. Matveev, VB, Salle, MA: Darboux Transformation and Soliton. Springer, Berlin (1991)

5. Hirota, R: The Direct Method in Soliton Theory. Cambridge University Press, Cambridge (2004)

6. Wang, $M, L i, X, Z$ hang, J: The $\left(G^{\prime} / G\right)$-expansion method and travelling wave solutions of nonlinear evolution equations in mathematical physics. Phys. Lett. A 372, 417-423 (2008)

7. Lu, DC: Jacobi elliptic functions solutions for two variant Boussinesq equations. Chaos Solitons Fractals 24, 1373-1385 (2005)

8. Yan, ZY: Abundant families of Jacobi elliptic functions of the $(2+1)$ dimensional integrable Davey-Stewartson-type equation via a new method. Chaos Solitons Fractals 18, 299-309 (2003)

9. Ablowitz, MJ, Clarkson, PA: Nonlinear Evolution Equations and Inverse Scattering. Cambridge University Press, Cambridge (1991)

10. Wang, M, Zhou, Y, Li, Z: Application of a homogeneous balance method to exact solutions of nonlinear equations in mathematical physics. Phys. Lett. A 216,67-75 (1996)

11. Gu, CH: Soliton Theory and Its Application. Zhejiang Science and Technology Press, Zhejiang (1990)

12. Wang, M, Li, X: Extended F-expansion and periodic wave solutions for the generalized Zakharov equations. Phys. Lett. A 343, 48-54 (2005)

13. Zhang, S: Application of Exp-function method to high-dimensional nonlinear evolution equation. Chaos Solitons Fractals 38, 270-276 (2008)

14. Ma, WX, Huang, T, Zhang, Y: A multiple exp-function method for nonlinear differential equations and its applications. Phys. Scr. 82, 065003 (2010)

15. Lou, SY, Lu, JZ: Special solutions from variable separation approach: Davey-Stewartson equation. J. Phys. A, Math. Gen 29, 4209-4215 (1996)

16. Wazwaz, AM: The tanh and sine-cosine method for compact and noncompact solutions of nonlinear Klein Gordon equation. Appl. Math. Comput. 167, 1179-1195 (2005)

17. Yan, ZY: The new tri-function method to multiple exact solutions of nonlinear wave equations. Phys. Scr. 78, 035001 (2008)

18. Yan, ZY: Periodic, solitary and rational wave solutions of the $3 D$ extended quantum Zakharov-Kuznetsov equation in dense quantum plasmas. Phys. Lett. A 373, 2432-2437 (2009)

19. Bluman, GW, Kumei, S: Symmetries and Differential Equations. Applied Mathematical Sciences, vol. 81. Springer, New York (1989)

20. Olver, PJ: Applications of Lie Groups to Differential Equations, 2nd. edn. Graduate Texts in Mathematics, vol. 107. Springer, Berlin (1993)

21. Ovsiannikov, LV: Group Analysis of Differential Equations. Academic Press, New York (1982). (English translation by WF Ames)

22. Ibragimov, NH: CRC Handbook of Lie Group Analysis of Differential Equations, vol. I. CRC Press, Boca Raton (1993)

23. Ibragimov, NH: CRC Handbook of Lie Group Analysis of Differential Equations, vol. II. CRC Press, Boca Raton (1994)

24. Ibragimov, NH: CRC Handbook of Lie Group Analysis of Differential Equations, vol. III. CRC Press, Boca Raton (1995)

25. Adem, KR, Khalique, CM: Exact solutions and conservation laws of a $(2+1)$-dimensional nonlinear KP-BBM equation. Abstr. Appl. Anal. 2013, Article ID 791863 (2013). doi:10.1155/2013/791863

26. Drinfeld, VG, Sokolov, V: Equations of Korteweg-de Vries type and simple Lie algebras. Dokl. Akad. Nauk SSSR 258, 11-16 (1981)

27. Satsuma, J, Hirota, R: A coupled KdV equation is one of the four-reduction of the KP hierarchy. J. Phys. Soc. Jpn. 51 3390-3397 (1982)

28. Gürses, M, Karasu, A: Integrable KdV systems: recursion operators of degree four. Phys. Lett. A 251, $247-249$ (1999)

29. Wazwaz, AM: The Cole-Hopf transformation and multiple soliton solutions for the integrable sixth-order Drinfeld-Sokolov-Satsuma-Hirota equation. Appl. Math. Comput. 207, 248-255 (2009)

30. Zheng, B: Travelling wave solutions of two nonlinear evolution equations by using the $\left(G^{\prime} / G\right)$-expansion method. Appl. Math. Comput. 217, 5743-5753 (2011) 
31. Kudryashov, NA: Simplest equation method to look for exact solutions of nonlinear differential equations. Chaos Solitons Fractals 24, 1217-1231 (2005)

32. Gradshteyn, IS, Ryzhik, IM: Table of Integrals, Series, and Products, 7th edn. Academic Press, New York (2007)

33. Ibragimov, NH: A new conservation theorem. J. Math. Anal. Appl. 333, 311-328 (2007)

34. Anco, SC, Bluman, GW: Direct construction method for conservation laws of partial differential equations. Part I: examples of conservation law classifications. Eur. J. Appl. Math. 13, 545-566 (2002)

35. Sjoberg, A: On double reductions from symmetries and conservation laws. Nonlinear Anal., Real World Appl. 10 3471-3477 (2009)

36. Muatjetjeja, B, Khalique, CM: Lie group classification for a generalised coupled Lane-Emden system in dimension one East Asian J. Appl. Math. 4(4), 301-311 (2014)

37. Adem, AR, Khalique, CM: On the solutions and conservation laws of a coupled KdV system. Appl. Math. Comput. 219 959-969 (2012)

doi:10.1186/s13661-014-0248-6

Cite this article as: Adem and Khalique: On the solutions and conservation laws of the coupled

Drinfeld-Sokolov-Satsuma-Hirota system. Boundary Value Problems 2014 2014:248.

\section{Submit your manuscript to a SpringerOpen ${ }^{\odot}$ journal and benefit from:}

- Convenient online submission

- Rigorous peer review

- Immediate publication on acceptance

Open access: articles freely available online

High visibility within the field

- Retaining the copyright to your article 